\title{
Insurance covered and non-covered complementary and alternative medicine utilisation among adults in Taiwan
}

\author{
Shu-Fang Shih ${ }^{\mathrm{a}, \mathrm{b}}$, Chih-Yin Lew-Ting ${ }^{\mathrm{b}, *}$, Hsing-Yi Chang ${ }^{\mathrm{a}}$, Ken N. Kuo ${ }^{\mathrm{a}}$ \\ ${ }^{a}$ Center for Health Policy Research and Development, National Health Research Institutes, No. 35, Keyan Rd., Zhunan Town, Miaoli County 35053, Taiwan \\ ${ }^{\mathrm{b}}$ Institute of Health Policy and Management, College of Public Health, National Taiwan University, No. 17, Xu-Zhou Rd., Taipei 10020, Taiwan
}

\section{A R T I C L E I N F O}

\section{Article history:}

Available online 22 July 2008

\section{Keywords:}

Taiwan

Complementary and alternative medicine (CAM)

Traditional chinese medicine

National Health Insurance

Help-seeking

\begin{abstract}
A B S T R A C T
Complementary and alternative medicine (CAM) has been characterized by prevalence, cost, and patterns of use. Factors determining its utilization have also been analyzed, either generally or for specific diseases, but few studies have considered the determinants of its frequency of use. Taiwan's pluralistic health care system and comprehensive insurance program covering Western medicine and traditional Chinese medicine (TCM) provide an interesting case to explore what forms of CAM people use, why and how often they use them. By using Taiwan's 2001 National Health Interview Survey and linking it with National Health Insurance (NHI) claims data, this study aims to investigate the sociodemographic, economic, behavioral and health determinants of use and frequency of use for both non-covered and covered CAM in Taiwan. The former consists of services practiced by non-regulated or non-NHI contracted practitioners and the latter includes those TCM covered by NHI. Our study showed that the determinants of using non-covered and covered CAM differ with respect to socio-demographic and behavioral factors, and health needs. Our evidence suggests that future CAM research must delineate between use and frequency of use to better understand the underlying factors contributing to initiation and continuity of CAM use.
\end{abstract}

(c) 2008 Elsevier Ltd. All rights reserved.

\section{Introduction}

The rise of interest in Complementary and Alternative Medicine (CAM) research may come from either an awareness of the rising popularity of non-orthodox therapies, a concern over the dominance and monopoly of the biomedicine (Lew-Ting, 2003), or the reimbursement issue of these services. Previous studies have reported the prevalence, cost, and patterns of CAM use (Eisenberg et al., 1993; Ernst, 2000; Harris \& Rees, 2000; Ni, Simile, \& Hardy, 2002; Thomas \& Coleman, 2004). Some have also investigated the factors associated with its use either generally or for specific diseases (Verhoef, Balneaves, Boon, \&

\footnotetext{
* Corresponding author. Tel.: +886 23322 8058; fax: +886 22351 5509.

E-mail addresses: annsshih@nhri.org.tw (S.-F. Shih), chihyin@ha.mc. ntu.edu.tw (C.-Y. Lew-Ting), hsingyi@nhri.org.tw (H.-Y. Chang), kennank@ nhri.org.tw (K.N. Kuo)
}

Vroegindewey, 2005; Maskarinec, Shumay, Kakai, \& Gotey, 2000; Garrow \& Egede, 2006; Grzywacz et al., 2006). However, from the health policy perspective, a study of whether these treatment modalities are used or not seems too naïve. In addition, how often people use them and why, may be more important because they can help us to better identify the needs of the users on a long-term basis.

Taiwan's medical pluralism is characterized by a dual professional system consisting of two great medical traditions: Traditional Chinese medicine (TCM) and Western medicine (Kleinman, 1980). Both are covered under Taiwan's National Health Insurance (NHI). However, since Western medicine still dominates health care resources (Chi, 1994) and political jurisdiction (Lew-Ting, 2003), TCM is still regarded as CAM. Coverage of TCM under the NHI includes Chinese medicines derived from traditional medicinal herbs, acupuncture, moxibustion, and traumatology manipulative therapy. TCM services covered by 
$\mathrm{NHI}$ are called covered CAM in this study. Outside the NHI, also part of the broader health care system (Kleinman, 1980), are some TCM treatments mentioned above practiced by non-NHI contracted providers and other CAM modalities of healing art such as folk remedies or spiritual healing etc. These are called non-covered CAM, which are less examined in Taiwan because it is usually considered not legitimate enough for policy research. With so many health care choices, Taiwan provides an interesting case to investigate people's healthcare-seeking behavior regarding both covered and non-covered CAMs.

Linking Taiwan's 2001 National Health Interview Survey (NHIS) with NHI claims data, this study describes the prevalence, types and patterns of use for both non-covered CAM and covered CAM and investigates socio-demographic, economic, behavioral and health determinants of use and frequency of use for both non-covered and covered CAM in Taiwan. Results from our study not only suggests that delineating between use and frequency of use can help to better understand the underlying motivations for CAM use but also provide more insights of the health needs of the people and healthcare-seeking behavior within and outside the health insurance system.

\section{Methods}

\section{Data source}

This study uses two data sets: the 2001 NHIS and 2001 NHI claims data. The 2001 NHIS collected health, health care, and socio-demographic information from a nationally representative sample of Taiwan's non-institutionalized population (Shih et al., 2003). Survey data were linked to the $2001 \mathrm{NHI}$ claims data for those participants who signed the informed consent allowing the linking of their NHI data. The utilization of covered CAM was drawn from the NHI claims data. We excluded the people with incomplete information. Only those people who are 20 years and older were included for their independent healthcare-seeking behavior. Therefore, the final samples consist of 10,600 and 9187 adults for the multivariate analyses of noncovered and covered CAM, respectively.

\section{Measures}

In this study, CAM is defined as treatment modalities that are not fully recognized by Western medicine in Taiwan such as TCM and other treatment modalities practiced by non-qualified or non-regulated practitioners or practitioners in folk sector, including both sacred and secular healing modalities (Kleinman, 1980). We classified CAM into "covered CAM" and "non-covered CAM" categories based on whether the modalities are practiced by practitioners with official licenses or the practitioners are NHI contracted providers. Taiwan's NHI covers TCM including traditional Chinese medicines derived from medicinal herbs, acupuncture, moxibustion, and traumatology manipulative therapy. However, some TCM services provided by non-NHI contracted or non-qualified practitioners are defined as non-covered CAM in this study. Information about utilization of covered CAM was drawn from the insurance claims data for each individual. The advantage of using linked data is that it provides clear and reliable records of which insurance covered CAM services the participants had used and how often they sought those services. It also reduced some possible recall bias in answering survey questions on past utilization.

The information about non-covered CAM was mainly drawn from the survey data. In the 2001 survey, respondents were asked to answer the question: "In the last one month, have you ever undergone any of therapies in this list in any non-medical setting?" The interviewer then read from a list covering the following seven CAMs: (1) shou-jing, a process in which a "demon" is compelled to leave a person who is physically or emotionally upset by an actual or imagined event; (2) ciou shen wun bu, a process in which the patient either draws lots from a bundle of bamboo sticks with special instructions or prays which is interpreted by an intermediary to ask for divine advice to treat physical or mental maladies; (3) siiang hui, eating the ash of temple incense; (4) scrapping, a process in which the skin is scrapped with a thin, flat object to unblock clogged energy in the body; (5) cupping, a process in which a suction cup is placed on a body part to suck out unclean energy; (6) bodywork, e.g., tuei-na massage and chiropractic care; and (7) bone setting and die-da sun shang, processes in which bones or ligaments are manipulated to treat musculoskeletal problems. In addition, the participants were asked an open-ended question regarding whether they had used any other similar therapies during the month leading up to the interview. In answering this question, additional CAMs were reported such as acupuncture, qigong (a type of relaxing, meditative body/mind/ spirit exercise), bloodletting, foot massage, the drinking of water blessed by a psychic, fortune changing (a process in which things are done or items are worn to change ones fate or reduce unlucky influences), prayers for divine intervention or advice and drawing from a bundle of sticks inscribed with various instructions on how to solve one's problems. If respondents reported having used any of these therapies, they were then asked how frequent in the past month they used them, why did they seek those types of therapy and how much they had spent on their latest therapeutic session.

A total of 14 types of non-covered CAM was reported as above. These modalities were further classified into 6 domains based on their nature and functions: (1) spiritual healing or healing involved supernatural forces (anything including rituals); (2) folk remedies (anything applied to or remove from the body using some accessories to relieve stagnation), (3) chiropractic care and massage (anything involving a practitioner manipulating the subject's body); (4) osteopathy (traumatology manipulative therapies): bone setting and die-da sun shang; (5) acupuncture by non-NHI contracted practitioners; and (6) qigong (any type of meditation or exercise).

We used 36-Item Short-Form Health Survey Taiwan version 1.0 (SF-36) to investigate the associations between the multi-dimensional health status and CAM use so that we could observe more specific dimensions associated with CAM use. The 8 dimensions covered by SF-36 include physical functioning, role limitation-physical, role 
limitation-emotional, mental health, social functioning, bodily pain, vitality, and general health. To take into account Taiwan's pluralisms in health system, we also constructed a variable called typical healthcare-seeking behavior from the open-ended question in the survey: "What do you usually do when you feel ill?" Most participants reported two behaviors. We used this question to categorize people into 4 types: (1) people seeking Western medicine only, (2) people seeking both Western medicine and others, (3) people who seek TCM and therapies others except Western medicine; and (4) other combinations of care. Other variables found to be associated with CAM use in previous studies such as age, gender, average annual household income, education level, ethnicity, marital status, number of having certain chronic conditions, religion, residential area, urbanization level and unhealthy lifestyle behaviors (such as smoking, drinking or betel nut chewing) were included in this study.

\section{Statistical analysis}

We used a two-part model to distinguish the factors related to use and use frequency for both non-covered and covered CAM. In the first part, we used logistic regression to analyze the factors associated with use of two sets of CAMs. In the second part, due to the over-dispersion of the number of visits to non-covered CAM and covered TCM, we used a negative binomial model to analyze factors associated with use frequency (Mullahy, 1986). Robust estimation was applied to take into account the heterogeneity.

\section{Results}

\section{Profile of CAM use}

Table 1 presents the prevalence rate and the number of visits per month for covered and non-covered CAM. In the survey, $6.05 \%$ of the respondents reported using at least one type of non-covered CAM in the month prior to the survey. The most frequently used were chiropractic care and massage (44.70\%), followed by folk remedies (35.63\%), spiritual healing (23.39\%), and bone setting and die-da sun shang (10.05\%). The average number of monthly visits per user

\section{Table 1}

Prevalence and average number of visits per month for covered and noncovered CAM

\begin{tabular}{lll}
\hline Type of CAMs & $\begin{array}{l}\text { Prevalence } \\
\text { rate (\%) }\end{array}$ & $\begin{array}{l}\text { Average number } \\
\text { of visits }\end{array}$ \\
\hline $\begin{array}{l}\text { Non-covered CAMs } \\
\text { (1) Spiritual healing or healing }\end{array}$ & 6.05 & 3.26 \\
$\quad$ involved supernatural forces & 1.41 & 1.44 \\
(2) Folk remedies & 2.16 & 2.51 \\
(3) Chiropractic care and massage & 2.70 & 3.52 \\
(4) Osteopathy & 0.61 & 4.02 \\
(5) Acupuncture & 0.19 & 2.39 \\
(6) Qigong & 0.01 & 1.50 \\
Covered CAM & 6.67 & 2.59 \\
(1) Chinese medicines derived from & 4.95 & 2.47 \\
$\quad$ traditional medicinal herbs & & \\
(2) Aupuncture, moxibustion, & 2.25 & 2.25 \\
$\quad$ traumatology manipulative therapy & & \\
\hline
\end{tabular}

for all non-covered CAM was 3.26, the highest was for bone setting and die-da sun shang (4.02) and the lowest for spiritual healing (1.44). Others averaged between 2 and 4 visits. With regard to results from the claims data, $6.67 \%$ had ever used covered CAM one month before the interview. Under covered CAM, $4.95 \%$ of respondents had ever used traditional Chinese herbal medicine, and $2.25 \%$ ever used acupuncture, moxibustion, and traumatology manipulative therapy. Overall, the average number of visits to covered CAM was 2.59 per month. The average number of visits that traditional Chinese medicinal herbs were used was 2.47 while either acupuncture, moxibustion, or traumatology manipulative therapy had been used 2.25 times.

Eighty-one percent of those using non-covered CAM used a single type, $14.32 \%$ a combination of two types, and $4.48 \%$ three or more types. The most used combination of modalities was scraping and massage (23.66\%), and the second involved three modalities: cupping, chiropractic and massage (21.37\%). When considering users of noncovered CAM and covered CAM altogether, around $85.90 \%$ could be categorized as single type users and around $10.56 \%$ combination users. The most common combinations of the two were chiropractic care and massage with the covered CAM (41.25\%), followed by a combination with scraping with covered CAM (28.75\%) and cupping with covered CAM (23.75\%).

Based on self-reported data from the NHIS, people most often sought non-covered CAMs for aches (12.92\%), peace of mind (9.46\%), and unexplained symptoms (8.95\%), followed by heat exhaustion (6.90\%), lower back pain (5.33\%), insomnia and sleeping disorder (3.78\%), influenza (3.76\%), tiredness and exhaustion (3.68\%), and headaches (3.17\%); 4.65\% refused to express their reasons for using non-covered CAM. Those most likely not to give a reason were users of folk remedies (32.82\%) and spiritual healing (8.41\%).

Factors associated with use and use frequency of non-covered and covered CAM

Table 2 shows the results from multivariate analysis of the determinants of decision to use and frequency of use for both non-covered and covered CAMs. Most likely to use non-covered CAM were middle-age people, women, people with higher education levels, those following traditional religions or Buddhism, those with more unhealthy behaviors, those with chronic conditions, those lived in central area, mixed users of health care services, and those with bodily pain. Our results showed that factors associated with use of non-covered CAM were also found to be associated with frequency of use. However, other factors such as ethnicity, urbanization level, and other health dimensions also determined the frequency of usage. In particular, those who had better physical functioning, and less bodily pain were less likely to be frequent users while those with better social functioning were more likely to make more visits to practitioners of non-covered CAM.

Only age, residential area, typical healthcare-seeking behavior, and bodily pain were associated with the decision to use covered CAM. Those aged 20-24 or 35-64 years old, 
Table 2

Multivariate analysis of factors associated with use and use frequency of CAM

\begin{tabular}{|c|c|c|c|c|}
\hline \multirow[t]{3}{*}{ Characteristics } & \multicolumn{2}{|l|}{ Use of CAM } & \multicolumn{2}{|c|}{ Frequency of CAM use } \\
\hline & \multicolumn{2}{|l|}{ Odds ratio } & \multicolumn{2}{|l|}{ Coefficients } \\
\hline & Non-covered & Covered & Non-covered & Covered \\
\hline \multicolumn{5}{|l|}{ Age $($ ref $=65$ or over $)$} \\
\hline $20-24$ & 1.56 & $2.01^{* *}$ & $0.78^{* * *}$ & $0.62^{* *}$ \\
\hline $25-34$ & $1.90^{* *}$ & 1.51 & $0.87^{* * *}$ & $0.53^{* *}$ \\
\hline $35-49$ & $1.92^{* * *}$ & $1.50^{*}$ & $0.99^{* * *}$ & $0.44^{* *}$ \\
\hline $50-64$ & $1.66^{* *}$ & $1.59^{* *}$ & $0.99^{* * *}$ & $0.63^{* * *}$ \\
\hline \multicolumn{5}{|l|}{ Gender $($ ref = male $)$} \\
\hline Female & $1.80^{* * *}$ & 1.07 & $0.53^{* * *}$ & -0.03 \\
\hline \multicolumn{5}{|c|}{ Education (ref=elementary or illiteracy) } \\
\hline Junior High School & 0.97 & 1.19 & -0.10 & $0.26^{*}$ \\
\hline Senior High School & $1.44^{* *}$ & 0.97 & $0.41^{* * *}$ & 0.03 \\
\hline College or above & $1.47^{*}$ & 0.99 & $0.30^{* *}$ & 0.11 \\
\hline \multicolumn{5}{|c|}{ Ethnicity (ref = Mainland and others) } \\
\hline Fukianese & 1.10 & 1.19 & $0.39^{* * *}$ & $0.27^{*}$ \\
\hline Hakka & 1.10 & 1.39 & $0.60^{* * *}$ & $0.33^{*}$ \\
\hline Indigenous & 1.54 & 0.87 & $0.69 *$ & 0.40 \\
\hline \multicolumn{5}{|c|}{$\begin{array}{l}\text { Average annual } \\
\text { household income } \\
\quad \text { (ref = below } 30,000 \text { NT dollars) }\end{array}$} \\
\hline $30,000-49,999$ & 0.89 & 1.00 & -0.07 & -0.06 \\
\hline $50,000-69,999$ & 0.80 & 0.94 & -0.08 & -0.09 \\
\hline $70,000-99,999$ & 1.11 & 1.04 & 0.06 & -0.02 \\
\hline 100,000 or above & 1.05 & 1.14 & 0.16 & -0.04 \\
\hline \multicolumn{5}{|c|}{ Religion (ref = Christianity, } \\
\hline \multicolumn{5}{|c|}{ Catholicism or Moslemism) } \\
\hline No religion & 1.07 & 1.12 & -0.07 & 0.23 \\
\hline Traditional religions & $1.57^{*}$ & 1.14 & $0.40^{*}$ & 0.33 \\
\hline Buddhist & $1.81^{* *}$ & 1.26 & $0.79^{* * *}$ & $0.44^{*}$ \\
\hline \multicolumn{5}{|c|}{ Marital Status (ref = otherwise) } \\
\hline Married & 1.14 & 1.13 & 0.01 & 0.11 \\
\hline \multicolumn{5}{|c|}{ No. of unhealthy behavior $(r e f=n o)$} \\
\hline One & $1.51^{* * *}$ & 1.09 & $0.54^{* * *}$ & 0.00 \\
\hline Two or three & $1.99 *$ & 0.63 & $1.28^{* * *}$ & -0.26 \\
\hline \multicolumn{5}{|c|}{ Chronic condition $($ ref $=n o)$} \\
\hline Yes & $1.25^{*}$ & 1.12 & $0.31^{* * *}$ & 0.14 \\
\hline \multicolumn{5}{|c|}{ Urbanization level (ref = medium) } \\
\hline Highest & 1.20 & 1.00 & $0.25^{* *}$ & 0.07 \\
\hline High-medium & 1.15 & 1.08 & $0.20^{*}$ & 0.14 \\
\hline Lowest & 1.06 & 0.87 & $0.18^{*}$ & -0.06 \\
\hline \multicolumn{5}{|c|}{ Residential area $(r e f=$ Southern $)$} \\
\hline Northern & 1.19 & $0.79^{*}$ & 0.06 & -0.11 \\
\hline Central & $1.48^{* *}$ & $1.30^{*}$ & 0.10 & $0.29^{* *}$ \\
\hline Eastern & 0.95 & 0.80 & $-0.62^{* * *}$ & -0.23 \\
\hline \multicolumn{5}{|c|}{$\begin{array}{l}\text { Typical healthcare-seeking behavior } \\
\text { (ref = Western medicine only) }\end{array}$} \\
\hline Western and others & $1.59^{* * *}$ & $2.18^{* * *}$ & $0.55^{* * *}$ & $0.77^{* * *}$ \\
\hline $\begin{array}{l}\text { Traditional Chinese } \\
\text { medicine and others } \\
\text { except Western med }\end{array}$ & $2.53^{* * *}$ & $4.72^{* * *}$ & $1.27^{* * *}$ & $1.59^{* * *}$ \\
\hline Others & $1.53^{* *}$ & 0.76 & $0.70^{* * *}$ & -0.26 \\
\hline \multicolumn{5}{|c|}{ SF-36 health status measure } \\
\hline Physical functioning & 1.02 & 1.00 & $-0.08^{* * *}$ & -0.03 \\
\hline Role-physical & 0.98 & 1.00 & -0.01 & 0.00 \\
\hline Role-emotional & 0.99 & 0.99 & -0.01 & -0.01 \\
\hline Social functioning & 1.02 & 1.05 & $0.04^{*}$ & 0.04 \\
\hline Bodily pain & $0.76^{* * *}$ & $0.91^{* * *}$ & $-0.30^{* * *}$ & $-0.07^{* * *}$ \\
\hline Vitality & 1.03 & 1.05 & -0.01 & 0.02 \\
\hline Mental health & 1.00 & 0.98 & 0.00 & -0.01 \\
\hline General health & 0.98 & 0.97 & -0.01 & $-0.05^{*}$ \\
\hline
\end{tabular}

Note: ${ }^{*} p<0.05,{ }^{* *} p<0.01,{ }^{* * *} p<0.001$.

those living in central and northern Taiwan, mixed users of health care, and those with bodily pain were more likely to use covered CAM. Similar to the findings from non-covered CAM, these factors were not only associated with decision to use but also found to be associated with frequency of use. Other factors such as education, ethnicity, religion, and general health conditions were also found to be associated with frequency of covered CAM use. Those with junior 
high education level, those of Fukianese or Hakka heritage, those following Buddhism, those with worse general health were more likely to make frequent visits to providers of CAM covered by the NHI program.

\section{Discussion}

Using a national representative sample from household survey and insurance claims data, this study assesses the determinants of use and frequency of use for both noncovered and covered CAM. This study moves beyond investigating whether CAM is used or not, but instead also examines the determinants of use frequency in the pursuit of deeper understanding about CAM use. This study is based on the assumption that frequency implies continuity and might, therefore, indicate preference for or effectiveness of certain modalities. More predictors were found to be associated with use frequency of both non-covered and covered CAM, suggesting that further examining the frequency of CAM use may provide a more precise picture of the phenomena.

According to our study, although almost all people in Taiwan are covered by insurance, there is still a higher demand for non-covered rather than covered CAM. The greater number of visits to non-covered CAM could have been associated with the greater variety of non-covered CAM available and longer course of treatment for some modalities. Unlike previous studies, none of our noncovered CAM users complained about their negative experiences with conventional/orthodox medicine. They did state that there were some problems that health care providers within insurance system could not treat, showing their concerns about the efficacies of these non-covered CAM services. The phenomena that people combined care from non-covered and covered CAM providers shows that people in Taiwan not only recognize the limitations of services provided within the insurance system but also tend to use health services selectively and adaptively (Lew-Ting, 2005). Since Taiwan's Medical Care Act does not regard non-covered CAM services as invasive, non-covered CAM is not regulated by Taiwan's Department of Health. Because their efficacy is questionable and they are not regulated, there is a need for evaluation and control.

Although it is hard to compare our findings with previous studies due to different definition of CAMs, study design, and health system setting, our findings on the socio-demographic determinants of non-covered CAM use such as age, gender, and education were consistent with previous representative population studies in the United Kingdom (Thomas \& Coleman, 2004), United States (Eisenberg et al., 1993), and Australia (MacLennan, Myers, \& Taylor, 2006). However, as for covered CAM, among these variables, only age was associated with its use decision although the pattern of relationship is slightly different from that for non-covered CAM. Our study showed that women tended to use and visit more to non-covered CAM while this situation was not the case for covered CAM. This might be partly because women were more willing to adopt or try different kinds of care outside of the insurance system for treating their health problems, and partly because it is easier for women than men to access to non-covered CAM information through their social networks. In addition, educational background was not associated with covered CAM use, suggesting that access to covered CAM did not vary across people with different education levels. Unlike some previous studies, income was not associated with use or frequency of use whether the modalities were covered or not. There are two possible explanations. First, under the NHI, people, especially for low income groups, may have more available financial sources, which, in turn, lead to better access to non-covered CAM. The spillover effect of NHI allows people to consume resources across its boundary. Second, it is also likely that people are willing to pay as much as they can to cure their diseases outside the NHI because they may have experiences with the limitations of conventional medicine. The perceived inefficacy and deficiency of services under the $\mathrm{NHI}$ pushes people make alternative choices.

In addition to the socio-demographic factors mentioned above, health belief or behavioral characteristics of insurance covered and non-covered CAM users also varied. Given that there were various types of non-covered CAM and less diversified therapeutic modalities of covered CAM, variables as proxies for health beliefs such as religion or having unhealthy behaviors were found to be more associated with non-covered CAM use than covered CAM use. Those who practiced traditional religions or Buddhism were more likely to use non-covered CAM; this might be due to the nature or philosophy of some non-covered CAM. Supernatural powers and sacred deity inherent in these modalities seem more acceptable to these people. Our study also found that a greater number of unhealthy behaviors led to choose and use more non-covered CAM. This could be found among people who were younger and healthier and may not in need of health care services provided by the NHI system. They might seek for noncovered CAM as a type of preventive medicine to ward of illness and protect their health. Further investigation is needed to confirm this hypothesis. Due to lack of sufficient sample size for each type of non-covered CAM, we could not conduct multivariate analysis to identify factors associated with use of specific type of CAM. Efforts such as collecting more complete data set on various types of CAMs should be encouraged as suggested by Astin (2000).

Our findings confirmed the hypothesis that CAM use can be seen as being more compatible with the patients' spiritual/religious philosophy, or beliefs regarding the nature and meaning of health and illness. For two sets of CAMs, more frequent visits of CAM use by Fukianese and Hakka ethnic groups has illustrated a deep cultural and belief influences on the continuity of CAM use. Those who practiced Buddhism were more likely than those with other western religions to visit more to covered and non-covered CAM; this also highlights the important role of cultural and social factors. As for health needs, our study indicates that more health dimensions found to be associated with use frequency than with use decision for both two sets of CAMs and the dimensions differed for two decisions except bodily pain. Our study suggests that for both two sets of CAM, they were used as means to treat illness in order to restore health rather than to prevent diseases or maintain health, especially in the case of pain relief. That those who had 
better social functioning tended to use more non-covered CAM might be due to the influences by others or information from their social groups/network members (Honda \& Jacobson, 2005).

Overall, factors such as age, residential area, typical healthcare-seeking behavior and health status were found to be not only significantly associated with use but also with frequency of use for both non-covered and covered CAM, although the pattern of relationship was slightly different in some cases. Middle-age persons tended to choose non-covered CAM while those aged 20-24 and 50-64 tended to choose covered CAM. However, the tendency to be frequent users across age groups was slightly different from the tendency to be users for non-covered CAM while it was found to be similar for covered CAM. Residential area was associated with availability and accessibility to CAM use, which cannot be controlled in our study due to lack of data, however, high frequent use of covered CAM in central area might be related to higher density of Chinese medicine doctor (Lee, Chou, Chen, \& Chang, 2004). Typical healthcare-seeking behavior implied the usual care provider people chose and reflected the freedom of care seeking under Taiwan's NHI (Cheng, 2003). More frequent use of both two sets of CAM were only associated with mixing the traditional Chinese medicine with other types of services but not with mixing Western medicine and other services. Health needs remained statistically significantly associated with use and frequency of use for both two sets of CAMs. Of all health needs, body pain remained the only ailment to be highly associated with use and frequency of use for both covered and non-covered CAMs. According to a recent Taiwan study, musculoskeletal and neurologic disorders were two major diseases commonly treated with acupuncture covered by the NHI (Chen, Kung, Chen, \& Hwang, 2006). Therefore, further investigation might be needed to understand how people use services within and outside NHI system to treat pain-related conditions.

This study has three limitations. First, since types of non-covered CAM being used were self-reported, the prevalence might be underestimated. Most participants only thought of some local or folk therapies or modalities that have existed in Taiwan for a long time but not other types of modalities such as aromatherapy, homeopathy, or complementary nutrition. However, this might also indicate that those "foreign CAMs" or others to be considered as CAM in other countries might not be so popular or not be perceived by the respondents as CAM in year 2001. Second, due to lack of measures in the survey data, we were not be able to directly examine the influences of health beliefs and attitudes on CAM use. To capture these effects, factors such as education, religion or ethnicity were used as proxies instead. Third, we lost some participants who did not agree that survey and NHI data be linked. However, we do not believe that declining to give consent affected our results much although one study has shown that the elderly, the illiterate, those with lower income, and suburban area residents were more likely to decline consent (Huang, Shih, Chang, \& Chou, 2007).

In conclusion, our study indicates that covered and non-covered CAM users were characterized by different socio-demographic and behavior factors, depending on the contents of services provided. Notably, it has evidenced unmet health needs of people even though most of them are covered by the NHI. This is what policy makers should pay greater attention to while planning an efficient and quality health care system in a society like Taiwan. Finally, in terms of measurements of CAM use, our evidence suggests that future research must delineate between use and frequency of use to better understand the underlying factors contributing to initiation and continuity of CAM use.

\section{References}

Astin, J. A. (2000). The characteristics of CAM users: a complex picture. In M. Kelner, B. Wellman, B. Pescosolido, \& M. Saks (Eds.), Complementary and alternative medicine: Challenge and change (pp. 101-114). Amsterdam: Harwood Academic.

Chen, F.-P., Kung, Y.-Y., Chen, T.-J., \& Hwang, S.-J. (2006). Demographics and patterns of acupuncture use in the Chinese population: the Taiwan experience. The Journal of Alternative and Complementary Medicine, 12(4), 379-387.

Cheng, T.-M. (2003). Taiwan's new national health insurance program: genesis and experience so far. Health Affairs, 22(3), 61-76.

Chi, C. (1994). Integrating traditional medicine into modern health care systems: examining the role of Chinese medicine in Taiwan. Social Science \& Medicine, 39(3), 307-321.

Eisenberg, D. M., Kessler, R. C., Foster, C., Norlock, F. E., Calkins, D. R., \& Delbanco, T. L. (1993). Unconventional medicine in the United States prevalence, costs, and patterns of use. New England Journal of Medicine, 328(4), 246-252.

Ernst, E. (2000). Prevalence of use of complementary/alternative medicine: a systematic review. Bulletin of the World Health Organization, 78(2), 252-257.

Garrow, D., \& Egede, L. E. (2006). National patterns and correlates of complementary and alternative medicine use in adults with Diabetes. The Journal of Alternative and Complementary Medicine, 12(9), 895-902.

Grzywacz, J. G., Suerken, C. K., Quandt, S. A., Bell, R. A., Lang, W., \& Arcury, T. A. (2006). Older adults' use of complementary and alternative medicine for mental health: findings from the 2002 national health interview survey. The Journal of Alternative and Complementary Medicine, 12(5), 467-473.

Harris, P., \& Rees, R. (2000). The prevalence of complementary and alternative medicine use among the general population: a systematic review of the literature. Complementary Therapies in Medicine, 8 , 88-96.

Honda, K., \& Jacobson, J. S. (2005). Use of complementary and alternative medicine among United States adults: the influences of personality, coping strategies, and social support. Preventive Medicine, 40(1), 46-53.

Huang, N., Shih, S.-F., Chang, H.-Y., \& Chou, Y.-J. (2007). Record linkage research and informed consent: who consents? BMC Health Services Research, 7, 18.

Kleinman, A. (1980). Patients and healers in the context of culture: An exploration of the borderland between Anthropology, Medicine, and Psychiatry. Berkeley: University of California Press.

Lee, C.-H., Chou, Y.-J., Chen, L.-S., \& Chang, H.-J. (2004). Utilization of ambulatory Chinese medical services under the National Health Insurance in Taiwan. Taiwan Journal of Public Health, 23(2), 100-107.

Lew-Ting, C.-Y. (2003). Who uses non-biomedical, complementary and alternative health care? Sociodemographic undifferentiation and the effects of health needs. Taiwan Journal of Public Health, 22(3), 155-166.

Lew-Ting, C.-Y. (2005). Antibiomedicine belief and integrative health seeking in Taiwan. Social Science \& Medicine, 60(9), 2111-2116.

MacLennan, A. H., Myers, S. P., \& Taylor, A. W. (2006). The continuing use of complementary and alternative medicine in South Australia: costs and beliefs in 2004. Medical Journal of Australia, 184(1), 27-31.

Maskarinec, G., Shumay, D. M., Kakai, H., \& Gotay, C. C. (2000). Ethnic differences in complementary and alternative medicine use among cancer patients. Journal of Alternative and Complementary Medicine, 6(6), 531-538.

Mullahy, J. (1986). Specification and testing in some modified count data models. Journal of Econometrics, 33(3), 341-365. 
Ni, H., Simile, C., \& Hardy, A. M. (2002). Utilization of complementary and alternative medicine by United States adults. Results from the 1999 National Health Interview Survey. Medical Care, 40(4), 353-358.

Shih, Y.-T., Chang, H.-Y., Liu, J.-P., Lin, H.-S., Chang, M.-C., Chang, F.-C., et al. (2003). The design, contents, operation and the characteristics of the respondents of the 2001 National Health Interview Survey in Taiwan. Taiwan Journal of Public Health, 22(6), 419-430.
Thomas, K., \& Coleman, P. (2004). Use of complementary or alternative medicine in a general population in Great Britain. Results from the National Omnibus Survey. Journal of Public Health, 26(2), 152-157.

Verhoef, M. J., Balneaves, L. G., Boon, H. S., \& Vroegindewey, A. (2005). Reasons for and characteristics associated with complementary and alternative medicine use among adult cancer patients: a systematic review. Integrative Cancer Therapies, 4(4), 274-286. 